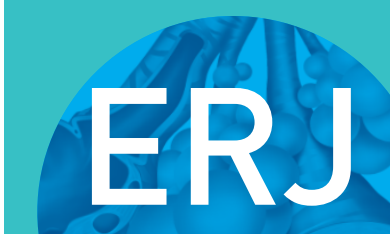

open research

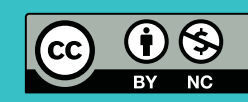

\title{
Factors that affect simulated driving in patients with obstructive sleep apnoea
}

\section{To the Editor:}

Patients with obstructive sleep apnoea syndrome (OSAS) are at increased risk of involvement in road traffic accidents (RTAs) [1]. Clinicians diagnosing OSAS need to advise patients about driving but there are no validated tools and no robust objective data about which factors are important [2]. There are position statements, based solely on expert opinion, from various professional bodies [3-6]. In general, they conclude that a patient with significant daytime sleepiness and a recent RTA or near miss attributable to sleepiness, fatigue or inattention, should be considered a high-risk driver [3-6]. In a recent survey using clinical vignettes, we have shown that there is considerable variability in the advice that patients are likely to receive [7]. This indicates that clinicians require more robust guidance.

We have previously established, using an advanced office personal computer (PC)-based driving simulator (Mini-UoLDS) [8], that it is possible to identify a subgroup of OSAS sufferers who perform significantly worse than others [9]. In contrast to other studies using simpler simulators in which, although patients with OSAS performed worse than controls, both groups still had large numbers of "off-road events" [10-12], 76\% of our patients were able to complete nearly an hour of "motorway" driving without incident [9]. Other studies using realistic simulation showed similar results [13]. More advanced PC-based simulators appear to be a credible test of real-life driving and may be useful in understanding what information is useful in assessing accident risk.

Patients attending our sleep clinic (St James' University Hospital, Leeds, UK) with a confirmed diagnosis of OSAS (apnoea-hypopnoea index (AHI) and/or oxygen desaturation index (ODI) (4\% dips in saturation) $>10$ per h) on a respiratory variable overnight sleep study (Embletta; ResMed Ltd, Abingdon, UK) or overnight oximetry were approached [9].

All completed a questionnaire about problems maintaining alertness while driving at each of six different times of day or while driving journeys of varying duration or on motorways, urban or rural roads. They were asked to indicate their chances of dozing or falling asleep in these situations using similar responses as for the Epworth Sleepiness Scale (ESS) score [14]. Their worst response determined the group into which the respondent was classified (e.g. a response of "slight chance" to any one of the 13 questions was taken as indicating that the patient was "slightly" sleepy while driving etc.). They were asked how long they would drive before taking a break, if in the last year they had ever nodded off whilst driving, driven over the rumble strip on the motorway or had a near miss, or whether they were involved in a RTA in the last 3 years.

All patients were asked to drive on a 90-km motorway simulation. "Failure" on the simulator was determined by predefined criteria. A detailed description of the simulator, its outcomes and measurements is available [9].

The demographic, clinical and polysomnographic characteristics and questionnaire responses were compared between two simulator outcome categories, "fail" and "not fail", using t-tests, Chi-squared tests or Fisher's exact tests. Binary logistic regression analysis was performed to explore whether a "fail" could be predicted from the demographic and clinical information and questionnaire responses. Receiver operating characteristic (ROC) curve analysis was performed to assess the predictive power of the regression equation.

133 patients were included (118 males; median (interquartile range) age 52 (44-60) years, body mass index 33 (30-37) $\mathrm{kg} \cdot \mathrm{m}^{-2}$, ESS score 12 (7-16), AHI 25 (13-42) per h and ODI 24 (13-44) per h). $32(24 \%)$ met criteria for "fail" [9].

The distribution and outcomes of demographic, clinical and polysomnographic characteristics and questionnaire responses are described in table 1 . Patients with a higher ESS score $(\mathrm{p}=0.04)$, those who admitted to having nodded off while driving $(\mathrm{p}=0.014)$ or taking a break within an hour of driving $(\mathrm{p}<0.001)$ were more likely to fail the simulator test. Patients who admitted to problems maintaining alertness while driving were also likely to fail and the likelihood increased from an odds ratio of 2.3 to 3.3 as the cut-off was moved from a "slight" to a "high" chance of feeling sleepy while driving. More severe OSA patients were more likely to fail, but this stopped short of statistical significance $(\mathrm{p}=0.08)$. Similarly, 


\begin{tabular}{|c|c|c|c|c|}
\hline Parameters & Fail & $\begin{array}{l}\text { Not } \\
\text { fail }\end{array}$ & p-value & $\begin{array}{l}\text { Chi-squared or Fisher's } \\
\text { exact test OR }(95 \% \mathrm{CI})\end{array}$ \\
\hline Patients n & 32 & 101 & & \\
\hline \multicolumn{5}{|l|}{ Patient parameters } \\
\hline Age years & $50 \pm 11$ & $52 \pm 10$ & 0.32 & \\
\hline $\mathrm{BMI} \mathrm{kg} \cdot \mathrm{m}^{-2}$ & $34 \pm 6.1$ & $35 \pm 6.7$ & 0.8 & \\
\hline Females & 7 (22) & $8(8)$ & 0.04 & $3.2(1-9.8)$ \\
\hline \multicolumn{5}{|l|}{ Clinical parameters } \\
\hline ESS score & $13 \pm 6$ & $11 \pm 5$ & 0.04 & \\
\hline $\mathrm{AHI}$ events per $\mathrm{h}$ & $34 \pm 24$ & $27 \pm 19$ & 0.15 & \\
\hline ODI events per $h$ & $38 \pm 27$ & $29 \pm 23$ & 0.08 & \\
\hline \multicolumn{5}{|l|}{ Driving parameters } \\
\hline Duration for which licence held years & $30 \pm 10$ & $32 \pm 11$ & 0.3 & \\
\hline Annual distance driven $>10000$ miles $^{\#}$ & $15(50)$ & $49(54)$ & 0.6 & $1.2(0.5-2.7)$ \\
\hline Predominantly local journeys & $17(55)$ & $55(55)$ & 0.85 & $0.92(0.4-2)$ \\
\hline HGV/PSV drivers & 1 (3) & $11(11)$ & 0.18 & $0.26(0.03-2.1)$ \\
\hline \multicolumn{5}{|l|}{ Driving questionnaire parameters } \\
\hline Taking a break at $<1 \mathrm{~h}$ during long journeys & $7(22)$ & $2(2)$ & $<0.001$ & $13(2.6-68)$ \\
\hline $\begin{array}{l}\text { Likelihood of feeling slightly sleepy } \\
\text { while driving }\end{array}$ & $25(80)$ & $65(65)$ & 0.09 & $2.3(0.86-6.1)$ \\
\hline $\begin{array}{l}\text { Likelihood of feeling moderately sleepy } \\
\text { while driving }\end{array}$ & $19(61)$ & $40(40)$ & 0.033 & $2.4(1.0-5.5)$ \\
\hline $\begin{array}{l}\text { Likelihood of feeling highly sleepy } \\
\text { while driving }\end{array}$ & 12 (39) & $16(16)$ & 0.006 & $3.3(1.3-8.2)$ \\
\hline Nodded off while driving in the last year & $14(48)$ & $24(24)$ & 0.014 & $2.9(1.1-4.0)$ \\
\hline Hit the rumble strip on motorway & $12(40)$ & 30 (32) & 0.33 & $1.4(0.6-3.2)$ \\
\hline Near misses in last 1 year & $8(27)$ & $20(20)$ & 0.43 & $1.4(0.56-3.7)$ \\
\hline Any accidents in last 3 years & 11 (37) & $22(22)$ & 0.09 & 2. $0(0.86-5.0)$ \\
\hline \multicolumn{5}{|l|}{ Simulator parameter } \\
\hline Study performed in the afternoon & $16(50)$ & $45(45)$ & 0.59 & $0.8(0.3-1.7)$ \\
\hline
\end{tabular}

Data are presented as mean \pm SD or $n(\%)$, unless otherwise stated. BMI: body mass index; ESS: Epworth Sleepiness Scale; AHI: apnoea-hypopnoea index; ODI: oxygen desaturation index; HGV: heavy goods vehicle; PSV: public service vehicle. ${ }^{\#}$ : $\sim 16000 \mathrm{~km}$.

there was a trend towards failing the simulator test in patients having had an accident in real life in the last 3 years (OR 2, p=0.09). Logistic regression analysis showed only admitting to taking a break less than an hour into long drives remained significant but the predictive power was not enough to be of any practical use by itself (ROC area under the curve 0.59, 95\% CI 0.47-0.71).

There is a relationship between "driving" on the Mini-UoLDS and patients' self-reported sleepiness and problems while driving in real life. A higher ODI was related to failure of the Mini-UoLDS but this was just short of statistical significance. This is in keeping with other studies [15-17]; these data suggest that patients should not be advised against driving solely on the basis of the severity of OSA alone, but it is a factor that should be taken into consideration.

A higher ESS score was related to an increased chance of failure of the Mini-UoLDS but the difference between the groups was small. Other studies have not shown a relationship between ESS score and accidents $[1,18]$. The ESS score in isolation should not be used to advise against driving.

MASA et al. [2] suggested that excessive sleepiness while driving, rather than sleepiness in general, may predict better which subjects with OSAS are at risk of RTAs. Our study confirms this. Patients with self-reported sleepiness while driving were more likely to fail. Admitting to taking a break less than an hour into long drives was strongly associated with a fail in the simulator, as was admitting to nodding off while driving. Nodding off at the wheel is a near miss that can only be attributed to sleepiness and is an all-or-nothing phenomenon, which is therefore more easy to define than "the likelihood of...". Patients who failed the simulator test showed an increased incidence of accidents in the previous 3 years, which approached statistical significance. It is not surprising that this relationship was not strong; accidents occur for a variety of reasons, of which driver sleepiness is only one. There may also be reasons other than OSAS for driver sleepiness. We relied on patients' reports and this has limitations. While we may have underestimated the proportion of patients with problems driving, this is likely to be an even bigger issue 
in everyday practice; we stressed to patients that no information from the study would be used to help inform decisions about driving. We think therefore that these responses are more likely to be honest than in everyday practice. In the absence of objective tests, clinicians will have to depend on the patients' accounts and this study therefore reflects the reality of everyday life. The rate of failures (25\%) of the Mini-UoLDS simulator is higher than the real-world accident rate in the general population of OSAS patients. However, ours was a subset with OSAS of sufficient severity to warrant treatment with continuous positive airway pressure; $25 \%$ of our patients admitted to an accident in the last 3 years. Our data, collected anonymously, may be more representative than data from official records: it is highly unlikely that patients will admit to accidents that have not happened. Our data are consistent with other studies; BARBE et al. [15] reported that $33 \%$ of patients with severe OSAS (AHI $58 \pm 3$ per h) had had an accident in the previous 3 years. MASA et al. [2] found that $33 \%$ of habitually sleepy patients with OSAS had had an accident in the previous 5 years.

This is the first study to provide objective data about what questions should be asked when advising a patient with OSAS about driving and will make future guidelines more evidence based. It is consistent with existing statements [3-6]. No single factor is sufficiently robust by itself, and the combination and contribution of each will have to be weighed by the clinician. These are the severity of the OSAS, sleepiness in general, sleepiness specifically while driving, a history of nodding off at the wheel, whether the patient needs to take a break less than an hour into a drive and any RTA in the previous 3 years. Most depend upon the patient being honest in their account. In the absence of a validated objective test, this will continue to be a problem for the clinician.

\section{Dipansu Ghosh ${ }^{1}$, Samantha L. Jamson ${ }^{2}$, Paul D. Baxter ${ }^{3}$ and Mark W. Elliott ${ }^{1}$}

${ }^{1}$ Dept of Respiratory Medicine, St James' University Hospital, Leeds, UK. ${ }^{2}$ Safety and Technology Group, Institute for Transport Studies, University of Leeds, Leeds, UK. ${ }^{3}$ Leeds Institute of Cardiovascular and Metabolic Medicine (LICAMM), Division for Epidemiology and Biostatistics, University of Leeds, Leeds, UK.

Correspondence: Mark W. Elliott, Dept of Respiratory Medicine, Sleep and Non-invasive Ventilation Services, St James's University Hospital, Beckett Street, Leeds, LS9 7TF, UK. E-mail: mark.elliott2@nhs.net

Received: July 032015 | Accepted after revision: Oct 262015

Acknowledgements: We are grateful to Michael Daly, Tony Horrobin and Hamish Jamson from the Institute for Transport Studies (University of Leeds, Leeds, UK) for their contribution to the development and running of the simulator. We are thankful to the contributions of research nurse Craig Armstrong, sleep physiologist Sue Watts, specialist nurses Martin Latham, Jampa Choedon, Mitchell Nix and Lisa Emmett, healthcare assistant Anne Kellett, research fellows Akshay Dwarakanath, Vinod Palissery and Audrey Rowe, and Susan Leigh from the Sleep Services administrative team (St James' University Hospital, Leeds, UK).

Conflict of interest: Disclosures can be found alongside this article at openres.ersjournals.com

@ERSpublications

Objective data for advising sleep apnoea sufferers whether they are at increased risk of an accident when driving http://ow.ly/TWPgm

Copyright $\odot$ ERS 2015. This article is open access and distributed under the terms of the Creative Commons Attribution Non-Commercial Licence 4.0.

\section{References}

1 Teran-Santos J, Jimenez-Gomez A, Cordero-Guevara J. The association between sleep apnea and the risk of traffic accidents. Cooperative Group Burgos-Santander. N Engl J Med 1999; 340: 847-851.

2 Masa JF, Rubio M, Findley LJ. Habitually sleepy drivers have a high frequency of automobile crashes associated with respiratory disorders during sleep. Am J Respir Crit Care Med 2000; 162: 1407-1412.

3 Austroads. Assessing Fitness to Drive for Commercial and Private Vehicle Drivers. Sydney, Austroads, 2014.

4 Ayas N, Skomro R, Blackman A, et al. Obstructive sleep apnea and driving: a Canadian Thoracic Society and Canadian Sleep Society position paper. Can Respir J 2014; 21: 114-123.

5 British Thoracic Society. Position Statement: Driving and Obstructive Sleep Apnoea (OSA)/Obstructive Sleep Apnoea Syndrome (OSAS). London, BTS, 2014.

6 Strohl KP, Brown DB, Collop N, et al. An official American Thoracic Society Clinical Practice Guideline: sleep apnea, sleepiness, and driving risk in noncommercial drivers. An update of a 1994 Statement. Am J Respir Crit Care Med 2013; 187: 1259-1266.

7 Dwarakanath A, Twiddy M, Ghosh D, et al. Variability in clinicians' opinions regarding fitness to drive in patients with obstructive sleep apnoea syndrome (OSAS). Thorax 2015; 70: 495-497.

8 Carsten OMJ, Groeger JA, Blana E, et al. Driver performance in the Engineering and Physical Sciences Research Council Driving Simulator: a validation study. Report No.: GR/K56162. Swindon, EPSRC, 1997. 
9 Ghosh D, Jamson SL, Baxter PD, et al. Continuous measures of driving performance on an advanced office-based driving simulator can be used to predict simulator task failure in patients with obstructive sleep apnoea syndrome. Thorax 2012; 67: 815-821.

10 Juniper M, Hack MA, George CF, et al. Steering simulation performance in patients with obstructive sleep apnoea and matched control subjects. Eur Respir J 2000; 15: 590-595.

11 Mazza S, Pepin JL, Naegele B, et al. Most obstructive sleep apnoea patients exhibit vigilance and attention deficits on an extended battery of tests. Eur Respir J 2005; 25: 75-80.

12 Turkington PM, Sircar M, Allgar V, et al. Relationship between obstructive sleep apnoea, driving simulator performance, and risk of road traffic accidents. Thorax 2001; 56: 800-805.

13 Vakulin A, Baulk SD, Catcheside PG, et al. Effects of alcohol and sleep restriction on simulated driving performance in untreated patients with obstructive sleep apnea. Ann Intern Med 2009; 151: 447-455.

14 Johns MW. A new method for measuring daytime sleepiness: the Epworth sleepiness scale. Sleep 1991; 14: 540-545.

15 Barbe,Pericas J, Munoz A, Findley L, et al. Automobile accidents in patients with sleep apnea syndrome. An epidemiological and mechanistic study. Am J Respir Crit Care Med 1998; 158: 18-22.

16 George CF, Boudreau AC, Smiley A. Simulated driving performance in patients with obstructive sleep apnea. Am J Respir Crit Care Med 1996; 154: 175-181.

17 Mulgrew AT, Nasvadi G, Butt A, et al. Risk and severity of motor vehicle crashes in patients with obstructive sleep apnoea/hypopnoea. Thorax 2008; 63: 536-541.

18 Young T, Blustein J, Finn L, et al. Sleep-disordered breathing and motor vehicle accidents in a population-based sample of employed adults. Sleep 1997; 20: 608-613. 